\title{
Gradhiva
}

GRADHI

Revue d'anthropologie et d'histoire des arts

16 | 2012

Chines, l'État au musée

\section{Le phénomène humain : l'avènement du réalisme}

\section{Gérard Lenclud}

\section{OpenEdition}

Journals

Édition électronique

URL : http://journals.openedition.org/gradhiva/2529

DOI : 10.4000/gradhiva.2529

ISSN : 1760-849X

\section{Éditeur}

Musée du quai Branly Jacques Chirac

\section{Édition imprimée}

Date de publication : 1 décembre 2012

Pagination : 240-253

ISBN : 978-2-35744-048-7

ISSN : 0764-8928

Référence électronique

Gérard Lenclud, «Le phénomène humain : l'avènement du réalisme », Gradhiva [En ligne], 16 | 2012,

mis en ligne le 01 décembre 2015, consulté le 21 septembre 2020. URL : http://

journals.openedition.org/gradhiva/2529; DOI : https://doi.org/10.4000/gradhiva.2529 


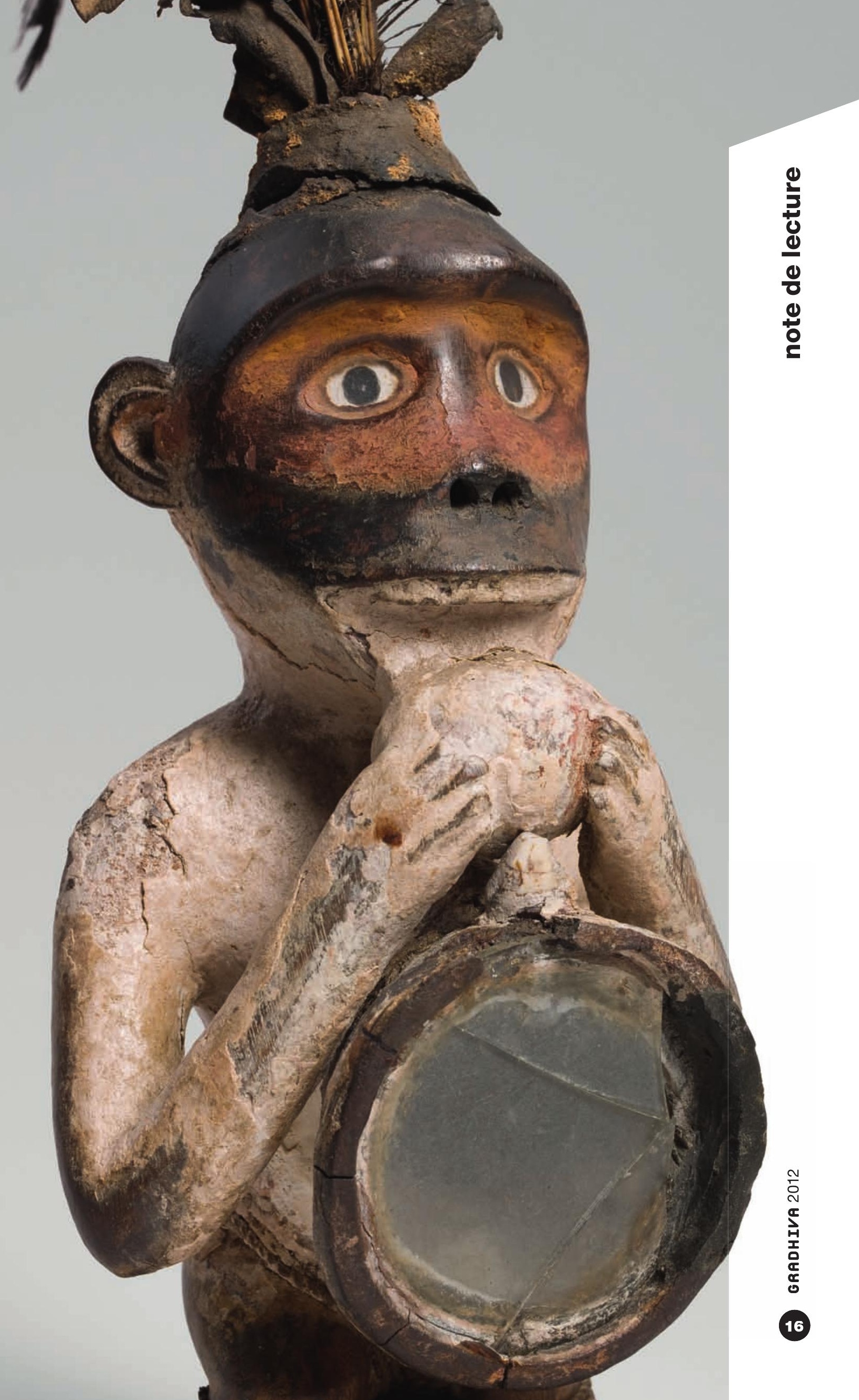




\section{Le phénomène humain}

\section{L'avènement du réalisme}

\section{par Gérard Lenclud}

Étienne Bimbenet, L'animal que je ne suis plus.

Paris, Gallimard («Folio Essais »), 2011, 512 p.

\section{"Le monde sensible est plus vieux que l'univers de la pensée. » \\ Maurice Merleau-Ponty}

Singulier tirage à la loterie des entreprises philosophiques, suis-je tenté de dire, que celle conduite par Étienne Bimbenet dans L'animal que je ne suis plus. Ce n'est évidemment pas parce que l'ouvrage traite de la question des frontières entre l'homme et l'animal; la différence anthropologique n'a jamais cessé, et pour cause, de figurer au programme de la philosophie. À supposer, d'ailleurs, qu'il soit licite, à la lumière des conquêtes de la zoologie, de l'éthologie et des sciences de l'évolution, d'évoquer l'animalité en général et de parler de l'animal sans user du pluriel. II semble que ce soit là l'une des multiples façons de sacrifier à l'anthropocentrisme et de se rendre intellectuellement complice de la "guerre sacrificielle», selon l'expression de Jacques Derrida, menée aux animaux, sinon depuis que l'homme est I'homme, car après tout l'homme n'est pas partout le même ni davantage le même de tout temps, du moins depuis que certains hommes en sont, à commencer par ceux que nous sommes. L'assignation indistincte d'animalité n'est sûrement pas pour rien dans la permission que nous nous accordons de considérer les animaux comme des objets, à consommer sans modération et sous d'innombrables formes. Ce n'est pas un hasard si, ici même, tous sont rangés dans la catégorie des «biens meubles» (article 528 du code pénal). Toutefois, le projet philosophique de Bimbenet justifie, comme on va le voir, le titre donné à son ouvrage.

Qu'il soit à placer à part, ce livre, ce n'est pas davantage, et précisément, parce que son auteur s'applique tout du long à repousser autant qu'il le peut la tentation anthropocentrique à laquelle, sous couvert d'humanisme, tant de discours philosophiques ont cédé par le passé. De nos jours, il est rare de revendiquer haut et fort le bien-fondé de l'attitude consistant à faire de l'homme la mesure de toute chose, dont par conséquent les animaux, dès lors chosifiés, et de décider à froid d'envisager la condition animale du seul point de vue des propriétés adjugées à la condition humaine, commuées dès lors en qualités absolues. Quel philosophe oserait aujourd'hui faire sienne cette attitude en toute connaissance de cause? L'adoption d'une perspective dite descendante, dans le vocabulaire des sciences cognitives, pour évaluer les capacités mentales des animaux, dans leur extrême diversité, ne saurait être assimilée au décret de principe selon lequel existerait une discontinuité parfaitement homogène entre n'importe quel représentant d'une espèce animale et l'homme. Une chose est de dire, par exemple, que les animaux ne pensent pas comme nous pensons, selon les critères en fonction desquels nous tendons à définir la pensée humaine; une autre est d'affirmer 


\section{Étienne Bimbenet L'animal que je ne suis plus}

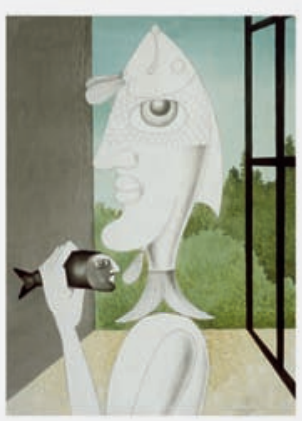

folioessais que les animaux ne disposent d'aucune forme de pensée, y compris ceux placés, par leur histoire évolutive, à faible distance généalogique de nous. Et ce décret, remarquons-le, heurte le sens commun. Qui s'applique à observer le regard d'un animal ne doute pas une seconde que cet animal dispose d'états de pensée.

Non, si l'ouvrage en question se signale à l'attention, ce n'est ni par son sujet, ni par son refus, soigneusement cultivé, de l'anthropocentrisme. C'est en raison de ses attendus initiaux et du choix opéré par Bimbenet, pour développer sa réflexion comparative sur l'homme et l'animal, de la méthode phénoménologique.

Le parti pris de départ, d'abord. II s'agit pour Bimbenet de parvenir à penser ensemble, et non séparément, encore moins concurremment, notre animalité d'hier et notre humanité présente. II rejette catégoriquement, en la matière, le principe du tiers exclu: ce n'est pas ceci ou cela, de deux choses l'une; ce doit être ceci et cela, l'une et l'autre choses à la fois. Animaux, nos lointains ancêtres le furent; nul ne peut en douter sérieusement. La conséquence est à en tirer, à savoir que c'est notre passé animal qui a fait de nous des hommes. Êtres humains, nous le sommes, c'est-à-dire plus des animaux, du moins dans une acception non classificatrice du terme; personne n'irait en disconvenir, en tout cas dans nos contrées culturelles. La conséquence est également à en tirer, concernant cette fois-ci notre rapport à ce passé: ce dernier aurait donc fabriqué un présent coupé de lui, ce qui ne veut pas dire que nous ne serions plus tributaires de notre histoire. Toujours est-il qu'en vertu des certitudes entourant notre statut d'êtres humains, la qualification de l'homme en tant qu'animal humain reste souvent au travers de la gorge. Pour sa part, Bimbenet la récuse sans la moindre hésitation. II considère en particulier que le «zoocentrisme» contemporain, sous-tendant le zèle égalitariste, consistant à proclamer peu ou prou que les animaux sont des êtres humains comme les autres témoigne d'une méconnaissance non seulement de l'homme mais bien aussi des animaux. Faudrait-il user du prétexte selon lequel ces derniers nous ressemblent en bien des points pour défendre la cause animale ${ }^{1}$ ? Pourquoi donc l'admission de la différence anthropologique devrait-elle fermer la porte au respect dû aux animaux? C'est tout le contraire, paraissent dicter les canons de l'éthique.

Bimbenet oppose radicalement deux perspectives dont chacune fait, selon lui, fi de l'autre. II y a, d'un côté, la perspective naturaliste. Elle oblige à prendre acte de nos origines animales et de l'unité structurelle du vivant. Or cette perspective en prend si bien acte qu'elle laisse entendre que l'homme est au bout du compte une variété d'animal parmi d'autres. Si l'espèce humaine est singulière, constituant à elle seule désormais le genre humain, elle l'est par définition de l'espèce: chaque espèce est celle qu'elle est et que n'est nulle autre, jusqu'à sa transformation ou son extinction. Aucune ne manque à la règle; si chacune est unique, aucune n'est exceptionnelle. Et, à l'image de toute espèce, la nôtre a acquis ses traits singuliers au terme d'une longue histoire naturelle, fort embrouillée, sans début véritablement assignable, entièrement tissée par le hasard et la contingence. Son advenue sur terre n'était pas programmée. Chacun sait, ou devrait savoir, 
que l'évolution est, sinon aveugle, du moins myope. Elle ne voit pas plus loin que ce qu'elle a sous les yeux; elle fait donc avec ce qu'elle trouve. Autant dire qu'elle bricole. Elle opère sur le moment, qui peut être long, et dans des lieux, sans prévoir ni, par conséquent, concevoir. Agent de pure exécution, nullement ingénieur attelé à une grande œuvre, sculpter, par exemple, sur le long terme notre humanité. La singularité d'Homo sapiens est le résultat d'une série d'événements parfaitement improbables, considérés rétrospectivement. Et à histoire naturelle, longtemps cantonnée dans le règne animal, singularité naturelle. Les sciences positives se doivent d'en rendre compte comme elles rendent compte de toute singularité spécifique au sein du vivant. Elle est à expliquer, sous-entendu pas à interpréter ou à comprendre. Un célèbre biologiste a écrit qu'il n'y avait rien dans l'aboutissement de notre histoire naturelle qui soit à comprendre.

Il y a là omission, selon Bimbenet, de quelque chose qui serait de l'ordre du fait. C'est un fait, éventuellement cousu de fil blanc mais non détachable de l'expérience humaine, institué si l'on veut, nullement brut, que les hommes, à tout le moins ceux composant notre humanité culturelle, exceptent leur état de la condition animale. Qu'il le veuille ou non, un homme ne voit pas un autre homme comme il voit un animal, si chargée d'empathie puisse être la manière dont il le voit. Même admettant pleinement son appartenance à l'empire du vivant, faisant de lui un être naturel, l'homme s'enferme spontanément, dit Bimbenet, dans les frontières de son humanité. En particulier, «il se fait un droit de son existence de fait» (p. 14). Tel qu'il se vit, tel qu'il attribue à ses semblables la qualité d'homme, faisant de chacun d'eux un alter ego et (en principe) un autrui, il élève cette qualité au rang de dignité. Nous, en tout cas, nous nous séparons de toutes les créatures qui ne sont pas nos semblables spécifiques. Et, note à juste titre Bimbenet, quand bien même nous accordons à des animaux sur le mode réfléchi la faculté de raisonner, l'aptitude à aménager politiquement la coexistence sociale, la capacité culturelle ou le sens moral, nous ne pouvons manquer, jusque dans les couloirs des colloques scientifiques, de pratiquer la restriction mentale: cela est vrai mais jusqu'à un certain point. Certes ils appliquent des raisonnements, ils se livrent à des manœuvres politiques, ils apprennent des comportements, ils font preuve de compassion, toutefois ce n'est sûrement pas comme nous. Entre les Pensées de Pascal et celles d'un chimpanzé, ou entre les tactiques d'un mâle dominant et les stratégies d'un prince éduqué par Machiavel, il y a tout de même un gouffre paraissant justifier cette séparation. Or l'établissement d'une coupure, fût-il opéré selon les modalités arbitraires de l'intime conviction, ne peut que participer d'un mouvement de «désanimalisation» de l'homme. Fiction peut-être, restauration métaphysique sans doute; il n'empêche que ce mouvement est à l'œuvre et qu'il travaillerait en profondeur l'humanité. Est-ce à bon droit épistémique que la perspective naturaliste se donne le luxe de l'ignorer ou de l'écarter?

Il y a, de l'autre côté, en position de face à face, la perspective humaniste. Point n'est besoin de beaucoup y insister. Elle accorde tout à l'homme, du moins tout ce qui compte à nos yeux d'hommes; à l'animal, elle concède le reste. II en résulte qu'elle consigne à l'être humain un statut ontologique quasiment hors nature. II serait, en somme, I'unique animal,
1. Bimbenet fait à bon droit justice de l'argument souvent avancé de la ressemblance génétique entre l'homme et le chimpanzé : ils ont $99 \%$ de leur ADN en commun. Comme le démontrerait aisément le généticien, l'ADN étant un code combinatoire discret, une différence de $1 \%$ est susceptible, en théorie, d'entraîner les mêmes conséquences qu'une différence de $100 \%$. II suffit, à cet égard, d'imaginer un texte écrit dont l'on changerait une seule lettre de chaque mot; il se pourrait qu'à l'arrivée l'on obtienne un texte différant du premier dans une proportion de $100 \%$. 
dans un certain sens, à n'en être pas un, dans un autre sens. Que pense l'humaniste, en effet, de l'animal? Prenons un exemple extrême. «Les bêtes n'ont pas d'esprit (mens), écrivait Descartes, et, par là, le nom d'âme (anima) est équivoque selon l'homme et selon les bêtes. "Dans cette perspective, la singularité de l'homme est d'un tout autre ordre que celle à lui assignée par la perspective naturaliste. Elle n'a rien à voir avec un ensemble ouvert de propriétés empiriquement dévolues à notre espèce, sur le modèle de celles échues à n'importe quelle espèce. Forçons le trait: cette singularité est un trésor, rassemblé dans une cassette sous le nom de propre absolu de l'homme. Notre singularité? Elle vient de nous, rien que de nous! L'humanité? Nous la valons bien! Nous sommes ceux que nous sommes, seuls en haut de l'échelle, fondés à nous contempler dans le miroir que nous nous tendons complaisamment et dans celui, tenu bien sûr par les mêmes mains, que nous tendraient nos voisins de règne. II va donc de soi que nous traitions de ces derniers en position de survol.

C'est au prix d'une mise entre parenthèses à tout le moins surprenante, à son tour, de la part de la perspective humaniste: celle du processus d'hominisation. Ainsi donc son déroulement, ayant mis en scène nombre d'espèces animales, aurait-il abouti à l'émergence d'un « produit ", l'homme, ne devant rien au processus. Ce serait comme si, toutes choses égales par ailleurs, un arbre, sous le prétexte qu'il développe sa majesté en altitude, devait être considéré comme ayant perdu tout contact avec ses racines, enfouies dans le sol. La différence anthropologique, mise sur le pavois, justifierait l'omission du fait évolutif. D'où le renvoi dans les oubliettes de l'animal que nous fûmes... et que nous ne sommes plus.

Selon Bimbenet, dont c'est l'attendu de départ, une vérité serait en suspens, hautement complexe à constituer, entre le naturalisme avéré par la science et l'humanisme professé, dit-il, en tout langage. «Nous visons mal notre humanité parce qu'elle s'annonce à mi-chemin entre deux perspectives adverses, et dont chacune, en réalité, paraît vraie jusqu'au bout » (p. 11).

Venons-en maintenant à ce qui fait l'originalité incontestable, dans ce domaine de la réflexion, de L'animal que je ne suis plus: le choix opéré par son auteur de la méthode phénoménologique pour comparer entre condition animale et humaine (et non, en principe, la condition animale à la condition humaine, cette dernière cessant alors d'être un terme de la comparaison pour en devenir l'instrument). Cette méthode permet, selon Bimbenet, et ici à coup sûr, d'éviter le Charybde du naturalisme, du moins dans sa version extrême dont les conclusions sont abusives, philosophiquement parlant. II reste que Bimbenet ne s'interdit aucunement de puiser à plein dans les diverses disciplines qui, à un titre ou à un autre, se penchent sur l'anthropogenèse: la paléoanthropologie, l'éthologie cognitive, plus généralement les sciences de la cognition, la primatologie, la psychologie comparée, la linguistique évolutionniste, y compris l'anthropologie sociale puisqu'il promeut certaines pages des Structures élémentaires de la parenté, traitant des rapports entre nature et culture, en grand récit de l'hominisation. C'est même tout le contraire. Ce philosophe, aussi philosophe qu'on peut l'être, évidemment familier de la tradition continentale mais aussi de la tradition anglo-saxonne comme de la philosophie de l'esprit, est allé 
aux textes scientifiques et à la documentation de laboratoire dont ils sont issus. Loin de les survoler ou d'y picorer, il en dissèque certains en détail. II y trouve une substantifique moelle, demeurée fréquemment inaperçue à l'extérieur des cercles disciplinaires, qu'il juge à l'occasion insuffisamment exploitée par ses découvreurs ou dont la portée véritable ne serait pas celle que ces derniers croient. C'est ainsi qu'il fait grand usage, par exemple, des données rassemblées sur le phénomène d'attention conjointe ou encore sur le geste de pointer du doigt ou de la main.

La méthode phénoménologique permet également, d'après Bimbenet, et ici cela peut être discuté, de passer au large du Scylla de l'humanisme, du moins de cet humanisme indifférent aux conditions de sa construction, soit fade de contenu puisque campant dans une humanité dessinée à partir de ses seules préconceptions, soit dépourvu de tout tranchant conceptuel en raison de son ignorance avouée du fait évolutif. La méthode phénoménologique autoriserait, en somme, à comprendre que l'homme, tout à la fois, vit avec son origine animale et, pourtant, sans elle. D'où le titre du livre, L'animal que je ne suis plus, expression à prendre de bout en bout le plus littéralement qui soit.

Choix de la méthode phénoménologique? Mieux vaudrait dire choix d'une certaine approche phénoménologique, celle exemplairement mise en œuvre par Maurice Merleau-Ponty dont l'auteur du livre est un spécialiste. Cette approche exclut, quant à elle, toute transcendance idéaliste ou métaphysique, dans un certain sens du mot, à la différence de ceux des phénoménologues ayant pris, selon la formule de Dominique Janicaud, un certain tournant théologique. (Notons, entre parenthèses, que les écrits de MerleauPonty ont influencé un courant des sciences cognitives, plus plurielles dans leur inspiration intellectuelle qu'il n'est souvent cru. Un ouvrage comme L'Inscription corporelle de l'esprit, paru en France en 1993, introduisant les notions d'action incarnée et d'enaction, entend tirer profit de l'idée, développée par Merleau-Ponty, d'une double corporéité: le corps comme structure vécue et comme domicile des mécanismes cognitifs ${ }^{2}$.)

Quoi qu'il en soit des divergences entre phénoménologues, le recours à la méthode phénoménologique pour comparer entre l'homme et l'animal peut légitimement surprendre comme peuvent étonner les louanges que lui tresse à l'avance l'auteur du livre. II s'agirait, en effet, d'une méthode admirable de précision, axiologiquement neutre qui plus est. Comme Bimbenet le rappelle, la méthode phénoménologique se caractérise par le rejet de principe du regard extérieur, ou de la position de surplomb, de règle dans l'enquête empirique et dans l'analyse causale, par la décision prise de ne rien avancer qui ne soit authentifié "en première personne», bref par la substitution du point de vue subjectif au dessein objectiviste. II semble que l'adoption de cette démarche et de son décret de méthode soit difficilement justifiable en regard des objectifs de connaissance visés par l'auteur, à savoir la relation à établir entre comportements animaux et humains. Attester "en première personne» ce que l'on en vient à dire d'un animal, à lui faire dire? En passer par le vécu personnel d'une créature non humaine pour comprendre, et comprendre, c'est de l'intérieur, ce que serait sa vie? Quel Je humain peut dire «je» à sa place? II faudrait admettre qu'un homme puisse accéder
2. Francesco Varela, Evan Thompson et Eleanor Rosch, L'Inscription corporelle de l'esprit. Sciences cognitives et expérience humaine, trad. de l'anglais par Véronique Havelange, Paris, Seuil, 1993. 


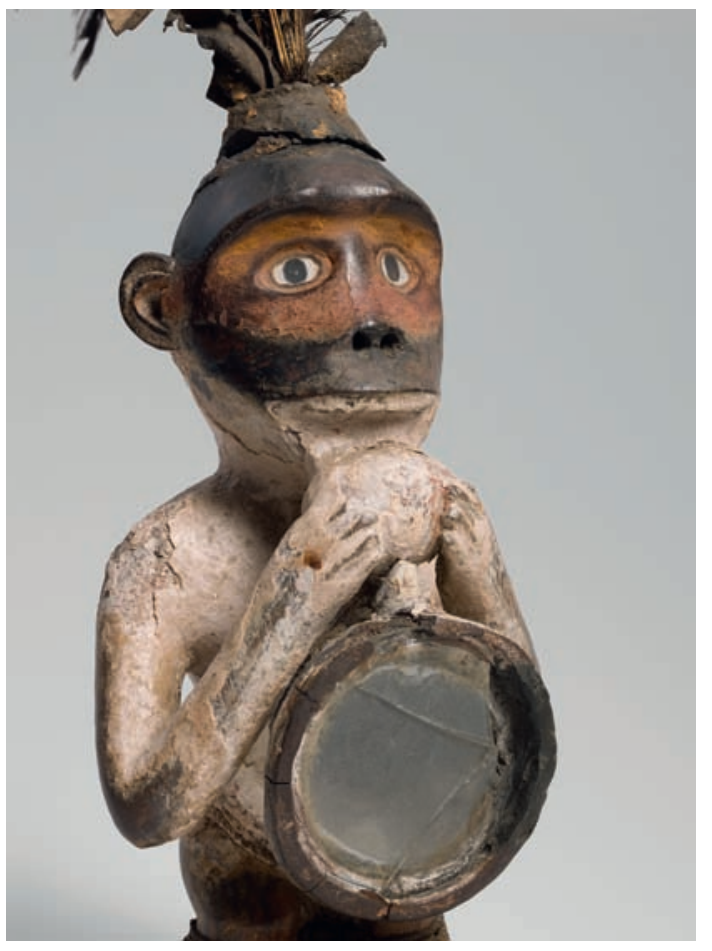

page 240 et ci-dessus

fig. 1

Statuette magique zoomorphe, Congo, av. 1892 (C) musée du quai Branly, photo Thierry Olivier, Michel Urtado. à l'effet que cela fait d'être une chauve-souris, s'orientant par écholocation, "comment c'est" de voir comme une grenouille ou d'être un chimpanzé en train d'épouiller son partenaire. Impossible, concède Bimbenet. II évoque à ce propos les recommandations, fort raisonnables à première vue, de l'adepte du «mystérianisme» selon qui la question de l'esprit animal est et sera à jamais un mystère, et contre qui Joëlle Proust a brillamment argumenté, à mon sens, pièces à l'appui ${ }^{3}$ dans son livre Comment l'esprit vient aux bêtes. Essai sur la représentation.

Impossible mais nécessaire, ajoute de suite Bimbenet. Ces sages recommandations laisseraient, en effet, de côté ce qui serait l'essentiel de la vie, de toute vie, à savoir que n'importe quel être vivant en est le sujet, dans l'acception phénoménologique du terme. Qu'il soit animal ou homme, l'être vivant est au cœur de sa vie, au centre de son rapport avec ce qui est son milieu de vie, d'une manière ressentie avant même d'être représentée, ou dûment cognitive. Nécessaire car l'explication "en troisième personne" ne saurait remplacer la compréhension «en première personne» ou, plutôt, en faire l'économie. C'est le cas pour l'homme, affirme bien sûr Bimbenet. Le phénoménologue détecte chez lui ce que le naturaliste se priverait de voir, la façon dont il expérimente son humanité dans un vécu d'évidence. Et ce vécu d'évidence, donc en toute subjectivité, ne se limite pas pour le phénoménologue aux qualia, ces maigres restes auxquels la philosophie de l'esprit, d'inspiration naturaliste, réduit la subjectivité humaine.

Fort bien en ce qui concerne l'homme, est tenté de concéder à son tour le sceptique, sachant au demeurant que le phénoménologue et le naturaliste cherchent à constituer des vérités d'un type très différent. Et, si rigoureuse puisse être la méthode phénoménologique, il demeure que sa rigueur s'évalue dans une autre monnaie que celle ayant cours dans les sciences de la vie. Mais, à coup sûr, c'est une autre affaire pour ce qui est de l'animal! Se glisser dans son vécu d'évidence? Expérimenter en soi la manière dont cet animal se vit au centre de sa vie? Comment Bimbenet procède-t-il pour conduire son entreprise, aussi impossible que nécessaire? Je doute qu'il soit d'accord avec la réponse cursive que je vais apporter à cette question mais je n'en ai pas d'autre disponible, sauf à l'avoir lu de travers.

D'abord, à l'exemple d'autres phénoménologues attachés à traiter de la vie, de la vie en général, Bimbenet se place, comme je l'ai déjà mentionné, au plus près de l'enquête scientifique. II entend soigneusement ce qu'elle dit de la vie. II n'est pas question pour lui d'en disqualifier a priori les enseignements. Après, mais après seulement, vient le moment de soumettre à critique les présupposés d'arrière-plan sur lesquels elle se construit, sa philosophie d'ombre, et de contester sa prétention à affirmer que c'est ainsi, et pas autrement, qu'il convient d'en déchiffrer les résultats. Bimbenet écoute donc avec la plus extrême attention l'éthologue ou le psychologue expérimental lui parler du rapport de l'animal au monde: comment il y adhère en le percevant, comment il l'investit en s'y comportant, comment il le fait sien en s'y liant, "corps et représentations", pour ne pas dire corps et âme. Puis il fait jouer l'aptitude proprement humaine à la variation, «eidétique" au sens où le phénoménologue évoque la réduction eidétique. II s'agit de cette capacité, détenue par nous, de multiplier les perspectives sur les choses, d'en imaginer 
de nouvelles, d'en emprunter et de les rapporter toutes les unes aux autres. Ce qui est, ou serait, nous sommes à même de l'envisager sous les modalités les plus diverses. Usant de cette aptitude, Bimbenet se lance alors dans une opération dont je suis tenté de dire qu'elle s'apparente à une traduction. En effet, me semble-t-il, il retranscrit «en première personne», celle de l'animal, une sélection de ce que l'enquête scientifique formule «à la troisième personne", celle de la science, donc des sciences de la vie. Option aventureuse, jurerait-on. Le phénoménologue ne se met-il pas alors à la place de l'animal, derrière ses yeux, en amont de ses actions? Mais Bimbenet se défend de mobiliser en l'occurrence une quelconque faculté d'empathie, un hypothétique pouvoir de projection. Non, maintient-il, le phénoménologue n'entre pas dans le corps animal, tel un corps étranger. Justement, ce corps animal, il ne lui est pas étranger ou pas si étranger que cela. La raison en est que, selon lui, l'homme vit «avec» l'animal comme il vit avec autrui et qu'il vit en sa compagnie dans un monde qui est là, pour l'homme, comme il est là, pour l'animal. C'est un monde commun, pour cause d'état de vie, à tous les êtres vivants. De là vient que Bimbenet s'autorise à affirmer, ou du moins à présumer, qu'il s'exprime du point de vue de nos origines animales, voire du point de vue de l'animal. La condition d'être vivant réunit condition animale et humaine avant que l'hominisation ne les sépare. Fort de cette conviction sur l'unité vécue du vivant, armé de cette traduction accomplie en vertu d'une disposition spécifiquement humaine à se déplacer d'une perspective à l'autre, Bimbenet s'estime alors en mesure de démontrer que les expériences animale et humaine ont quelque chose d'essentiellement différent. II juge être en position, nullement de surplomb, de faire voir et comme toucher du doigt, "en chair et en os ", la différence qualitative entre l'animal et nous, une différence radicale dans l'être-au-monde. Au lecteur de trancher sur le fait de savoir si la démarche phénoménologique est, dans ce cas précis, aussi rigoureuse et axiologiquement neutre que Bimbenet cherche à l'en persuader.

Au reste, ce qui importe est bien plutôt l'enseignement tiré par Bimbenet de sa comparaison entre expérience animale et humaine. Quelle est donc cette vérité attendant quelque part son dévoilement, au confluent en théorie introuvable entre le vrai naturaliste et humaniste? En quoi consiste au juste cette différence qualitative, ontologiquement déterminante, entre l'êtreau-monde de l'animal et de l'homme? On notera, à propos de cette vérité, que son contenu justifie entièrement ce qu'en annonce Bimbenet. Assurément, dit-il, «la phénoménologie ne pourvoira la science d'aucun fait nouveau»; elle n'est pas en position de le faire et, par conséquent, de le proclamer. Toutefois, ajoute-t-il immédiatement, "elle offre un nouveau point de vue sur le phénomène humain qui pourrait s'avérer parfaitement compatible avec celui de la science» (p. 42). Cette vérité réside dans ce point de vue. C'est un fait que ce dernier est nouveau en ce sens qu'il renverse la manière habituelle d'opposer l'animal à l'homme, le tenu pour vrai de l'humaniste, ou au contraire d'estomper cette opposition, le tenu pour vrai du naturaliste. Tout comme c'est un fait que ce point de vue ne contredit pas formellement les données rassemblées par les enquêtes empiriques si, en maints endroits, Bimbenet argumente contre les raisonnements conclusifs à l'œuvre dans l'analyse causale. Incontestablement inédit dans le contexte actuel, du moins - avouons-le - celui qui m'est le plus familier, étonnant à première
3. Joëlle Proust, Comment l'esprit vient aux bêtes. Essai sur la représentation. Paris, Gallimard ( NRF Essais »), 1997. 
vue dans sa formulation, compatible néanmoins avec la matière première des observations empiriques, il place à un autre endroit que celui ordinairement localisé le fossé creusé entre l'animal que nous fûmes et l'homme que nous sommes devenus. Cet endroit où le second aurait rompu les ponts avec le premier sans, pour autant, devoir renier ses origines, c'est là où, et quand, tard venu dans l'histoire de la vie, naît le réalisme.

L'expérience animale? Reprenant le fond des analyses déjà anciennes de Jacob von Uexküll, voici comment la définit Bimbenet: «L'animalité est subjectivité; il n'y a même de subjectivité qu'animale, l'animal est le seul vrai sujet» (p. 123). Sujet, il ne l'est certes pas au terme d'un acte de réflexion, ni ne l'est-il au sens où il se barricaderait à double tour dans les frontières de sa subjectivité, hermétique à tout ce qui n'est pas lui. Sujet, pas davantage ne l'est-il en ce que la satisfaction de ses besoins dirigerait ses comportements et qu'il serait tout entier à sa survie attaché. S'il est pur sujet, c'est parce que sa vie se déroule à l'état vécu dans un monde où n'est présent pour lui que ce qu'il y a mis. Son mode d'être-au-monde coïnciderait avec son mode d'êtreà-soi. Rien ne viendrait s'intercaler entre les deux. L'animal ne serait donc aucunement cet être purement fonctionnel, poussé par la nécessité, comme le penserait intuitivement l'homme. Il est cet être qui fait de celui qu'il est la mesure de toutes les choses dans le monde. En effet, elles n'existent pour lui qu'en tant qu'il les fait siennes. L'animal est donc la seule créature authentiquement perspectiviste: sa subjectivité est constituante du monde; elle l'institue.

La condition humaine? Elle est celle d'un être vivant qui, tout en éprouvant le monde au plus profond de lui, sans que jamais soient dénoués ses liens avec le sensible, place ce monde, dans le même temps, à distance de lui. Le monde est pour l'homme, à la différence de l'animal, autre chose dans son statut de monde que ce monde qu'il expérimente subjectivement. Il existe en soi, indépendamment de lui; ainsi ce dernier le ressent-il intérieurement. Certes les choses sont corrélatives de son corps connaissant mais cette corrélation n'est pas le tout de ces choses. L'hominisation consiste donc, selon Bimbenet, dans l'introduction sur terre du regard réaliste. L'être humain est cette créature, unique dans le règne animal, qui pose l'existence du monde extérieur et croit, mais c'est là une illusion (bien que Bimbenet ne l'affirme qu'entre les lignes), que le monde est là, ne devant rien au regard lancé sur lui. Contrairement à l'animal, lequel ne "croit» pas que le monde existe, plus exactement n'a pas à le croire, l'homme fait droit à la réalité du monde. II reconnaît son antériorité par rapport à lui; il se place sous son autorité en s'interrogeant sur ce qu'il est, sur ce que sont véritablement les choses, admettant par là même qu'il a affaire au monde et pas seulement à lui-même. "La transcendance du monde est une structure spécifiquement humaine de l'expérience, advenue une fois seulement dans l'histoire de la vie» (p. 146). Le réalisme serait notre "attitude naturelle» avec tout ce qu'elle implique d'ascendant consenti au monde.

Voilà qui va également à l'encontre de nos conceptions spontanées, ici en tout cas, d'un ou du privilège humain. En effet, celles-ci nous dictent l'idée que ce sont nous, les hommes, qui serions les seuls véritables sujets de nos vies, délivrés du poids écrasant de la soumission au monde, lot de l'animal. C'est peu ou prou l'idée de l'homme maître du monde 
comme de l'univers. Or, à suivre Bimbenet, il faudrait presque inverser la formule; c'est précisément la soumission réaliste au monde qui serait au cœur du destin humain. L'homme se plie au monde quand l'animal plie la réalité à sa subjectivité.

L'avènement du réalisme serait le ressort du grand partage à établir entre eux et nous. L'animal est sûr et certain du monde puisque ce dernier est, dans son entièreté, institué par lui. Son monde est une auberge espagnole. L'homme, pour sa part, "se fie» au monde puisque le monde, et non un monde, est là, toujours déjà là, auquel il se heurte. «Le réalisme est ce qui se vit, quand le réel est ce qui se voit» (p. 143, italiques dans le texte). Son attitude naturelle lui proscrit de confondre le monde avec ce qu'il s'en représente ou plus exactement selon Bimbenet avec ce qu'il en vit. Remarquons, à ce propos, l'écart considérable entre le point de vue du phénoménologue et celui du philosophe de l'esprit, d'inspiration naturaliste. En vertu de la pure subjectivité de l'animal, Bimbenet se doit de lui refuser toute aptitude à l'objectivité. Un philosophe de la cognition serait tenté de lui faire observer qu'il ne fait donc pas don à l'animal d'un mental, c'est-à-dire d'un esprit dans le cadre d'une conception représentationnelle de l'esprit, conception que Bimbenet rejette. Le philosophe de la cognition, pour sa part, accorde, sinon à l'animal, du moins à certaines catégories d'animaux, en fonction de leur histoire évolutive, cette aptitude à l'objectivité dès lors qu'ils se révèlent en mesure de dissocier leurs états représentationnels des choses et des situations que ces états représentent. Le monde ne serait donc pas, pour eux, épuisé dans l'expérience qu'ils s'en font. La dualité de langage philosophique entre le phénoménologue et le «cognitiviste» fait qu'au bout du compte l'animal du premier, tout un, n'est pas qualitativement identique à celui du second, soigneusement repéré en fonction de sa position dans l'histoire évolutive du vivant. L'homme non plus; on s'en doute.

Sous la plume du phénoménologue, le réalisme est une propriété phénoménale (vécue) de l'expérience humaine, pas une composante cognitive, du moins au premier chef. La «postulation» du monde comme unique monde est loin d'être d'ordre représentationnel, dans le langage dominant de la philosophie de l'esprit, à supposer encore que cette dernière songe à mobiliser, telle quelle, cette notion de réalisme.

La place me manque pour suivre dans le détail la démonstration qu'entend opérer Bimbenet de la validité de son point de vue, à savoir que le surgissement de l'attitude réaliste a fait le tout de la différence anthropologique. À cette fin, il se penche successivement sur la perception animale et humaine, sur la communication chez nos voisins de règne et le langage chez nous, sur le phénomène de l'attention conjointe en ce qu'il permettrait de discerner le gouffre, selon lui, entre le mode d'être à l'autre de l'animal et celui de l'homme. Ses analyses sont minutieuses, attentives aux données rassemblées dans les enquêtes empiriques mais en complète contradiction avec les attendus initiaux de la plupart de leurs auteurs et avec la philosophie générale qui en inspire les conclusions.

En effet, fidèle à la tradition phénoménologique, Bimbenet manifeste plus que de la méfiance à l'encontre du postulat «intellectualiste » à l'œuvre 
dans cette philosophie, découlant du «mentalisme» le plus souvent adopté; c'est bien plutôt un rejet de principe. II évoque à ce propos, dès le départ, un «mentalisme vieilli» (p. 37) et, plus loin, «les préjugés qui saturent, de manière hyper-cartésienne, le cognitivisme des années 1990 » (p. 348). À le lire, on ne peut que songer aux lignes inaugurales du dernier ouvrage achevé de son vivant par Merleau-Ponty, L'CEil et l'Esprit ${ }^{4}$. II y dénonce, en 1964, la science qui «manipule les choses et renonce à les habiter», portant à l'absolu «la situation de connaissance comme si tout ce qui fut ou est n'avait jamais été que pour entrer au laboratoire». II y proclame la nécessité de transformer, sinon l'activité scientifique, en tout cas certaine philosophie des sciences afin qu'elle se commue en véritable philosophie. Non, selon Bimbenet, le critère de la connaissance ne doit pas dominer la comparaison entre l'animal et l'homme, et donc servir à fonder l'entièreté de la différence anthropologique.

Faute de pouvoir entrer dans le cœur de ce gros ouvrage et donc de restituer les étapes de la polémique de méthode, au sens fort du terme, engagée par son auteur contre le «mentalisme», je me bornerai à introduire, sous forme simplifiée, deux points stratégiques de son argumentation. Le premier se trouve dans l'un des trois chapitres consacrés à la perception, celui intitulé «La perception humaine: la multiplicité perspective», situé dans le prolongement direct des écrits de Merleau-Ponty. II estime y livrer, dans ce domaine évidemment capital qu'est la perception pour comparer entre l'animal et l'homme, une attestation phénoménologique de la différence anthropologique, entraînée par l'avènement du réalisme. (On se souvient, au demeurant, que la multiplicité perspective à la disposition de l'être humain serait ce qui l'autorise à comprendre la perspective de l'animal, sinon à voir derrière ses yeux.) À l'évidence, tout être vivant voit d'un lieu. Pure subjectivité, subjectivité créatrice de son monde, l'animal est, pour sa part, rivé à son lieu. II ne peut voir que de là où il voit et de la manière immédiate dont il voit. II ne voit donc chaque chose, ou chaque événement, que sous l'aspect où il la fait sienne en faisant sien le monde tout entier. Bimbenet reprend ici un exemple choisi par Merleau-Ponty: celui de la branche d'arbre dont le chimpanzé se fait un instrument fonctionnel. Sitôt devenu bâton, perçu bâton et non "comme un" bâton, la branche d'arbre disparaîtrait de l'horizon, perceptivement vécu, du primate non humain. Elle s'évanouit dès lors que le bâton est là. En ce qui le concerne, l'homme n'est pas prisonnier du lieu, non purement géographique, d'où il voit. Ainsi, dans le cas de la branche utilisée en guise de bâton, verrait-il la chose, la même chose, sous deux aspects, "comme» une branche et "comme» un bâton, et sous bien d'autres encore. Cette chose, la même, est visible pour lui sous une pluralité de points de vue; et cette pluralité est commandée par l'attitude réaliste. Ici réside la différence extrême, selon Bimbenet, entre le perspectivisme de l'animal, parfaitement univoque puisque chaque chose est celle qu'elle est en vertu d'une seule perspective, la perspective possessive prise par l'animal, et le perspectivisme humain, se définissant par la capacité de l'homme à faire varier son point de vue sur chaque chose, visible dès lors sous une infinité d'aspects rassemblés dans une cohabitation rigoureuse et cohérente. C'est la même chose; le monde en impose la conviction, embrassé à partir de perspectives différentes. Bien que toujours d'un lieu, jamais délestée de son ancrage corporel, notre vision des choses du monde est donc une «vision à mille 
regards" (Merleau-Ponty), nullement captive du lieu, ainsi que l'est celle de l'animal. Et l'existence de ces mille regards, nous l'anticipons, ressentant en nous que le monde tel qu'il est, dans son objectivité de monde, est ouvert à une exploration sans fin, faisant par là même autorité sur nous et nous contraignant à s'y soumettre.

L'analyse de la multiplicité perspective chez l'homme trouve son complément obligé dans l'analyse de la capacité au langage; et ce complément est exemplairement illustré par le phénomène d'attention conjointe, significativement désigné sous l'expression de «partage du monde». En effet, le langage, que Bimbenet place à une distance sidérale de la communication animale, contrairement à la position (relativement) continuiste de la linguistique évolutionniste, et cela en raison de son caractère prédicatif - cette chose est ceci ou cela, comme ceci ou comme cela, comme ceci et comme cela - l'opposant à la dimension injonctive des signaux animaux, est le plus évident facteur de multiplication de la multiplicité perspective, lié tout autant à la comparution consentie devant le tribunal du monde. Le rapport à autrui tissé par le langage permet de lui emprunter son point de vue et d'envisager les choses du monde sous ce point de vue. Je peux les voir comme autrui les voit. Si l'animal fait siennes ces choses, je suis en mesure de faire mien ce point de vue. La vision humaine n'est jamais celle d'un être solitaire; elle est d'emblée établie dans un espace intersubjectif; elle se solidarise avec celle émanant d'autres corps qui connaissent. «La multiplicité perspective n'est pas le fait d'un être désincarné, mais l'exercice d'un regard partagé» (p. 197). Autrui est à la source vive du principe de variation de mes points de vue. Les mille regards de ma vision sont autant les siens que les miens.

C'est à la condition de se déprendre, tient à préciser Bimbenet, d'une certaine conception du rapport à autrui, trop abstraite, entendons: trop intellectualiste, faisant de lui un être à déchiffrer comme de l'extérieur, à la façon d'un problème à résoudre. II n'y aurait pas à pénétrer ses parois de chair pour parvenir à ce qu'il éprouve de l'intérieur. Celles-ci ne sont pas un obstacle à surmonter mais une voie d'accès direct à autrui. À cette occasion, et sans que je puisse m'étendre sur le sujet, Bimbenet tranche assez abruptement à propos des débats suscités par la théorie de l'esprit, cette faculté de prêter des états mentaux à autrui, et expédie en quelques lignes l'interprétation simulationniste des neurones miroirs. La théorie de l'esprit, telle qu'envisagée par la psychologie comparée, et ce modèle d'interprétation sacrifient trop, selon Bimbenet, à l'idée d'une séparation entre ce qui serait de l'ordre du Moi et du Toi.

Je ne vois guère d'autre conclusion possible à cette recension trop rapide d'un ouvrage aussi riche en développements que celle-ci: la recommandation de le lire. Cette lecture procurera sans doute à qui n'est pas philosophe, encore moins familier de la tradition phénoménologique, le plaisir rare qui est celui du dépaysement intellectuel. Elle amène en tout cas à faire varier ses propres perspectives sur la question de la différence anthropologique et peut-être conduira-t-elle le lecteur à interroger ses certitudes à ce propos, si d'aventure il en avait.
4. Maurice Merleau-Ponty,

L'CEil et l'Esprit (1964).

Paris, Gallimard

(«Folio Essais»), 1992. 


\section{ci-contre}

fig. 2

Quadrupède à tête anthropomorphe ammassalik, Inuit (C) musée du quai Branly, photo Thierry Olivier, Michel Urtado.
Puisque ces lignes sont écrites dans Gradhiva, cette recommandation s'adresse tout particulièrement aux anthropologues, mais sans oublier les spécialistes d'archéologie préhistorique. Ces derniers s'attachent, en effet, à extraire du sol et à sonder les témoignages de l'émergence sur terre de cet être qu'ils nomment "l'homme culturellement moderne" et que Bimbenet appellerait tout simplement l'Homme. Ne se pourrait-il qu'en lieu et place des traces de sa maîtrise du monde, ils découvrent en fait celles de l'avènement du réalisme? Homo culturalis serait ce premier animal admettant que la réalité lui impose sa loi. Quant aux anthropologues, ils trouveront matière à réfléchir et, selon toute probabilité, à contester les thèses présentées dans L'animal que je ne suis plus (sauf, à n'en pas douter, celles qui s'attaquent au cognitivisme). En deux mots, voici pourquoi, sachant qu'ils ont bien d'autres raisons de s'y intéresser. L'argumentation soutenue par Bimbenet participe de l'universalisme le plus radical qui soit. Tout relativisme en est exclu, à commencer, implicitement, par le relativisme culturel. C'est pourquoi, ici et là dans ma recension, j'ai été tenté de suggérer que peut-être fallait-il apporter quelques nuances dans ce portrait unitaire de l'homme. Cet homme s'enferme-t-il partout à l'identique dans les frontières de la stricte humanité, porteur de la même attitude réaliste envers le monde? Bimbenet en est si assuré - c'est nécessaire en un sens à sa démonstration - qu'il ne songe guère à se poser la question. Or les anthropologues ont dépeint des sociétés où les frontières au sein du vivant ne sont pas dressées aussi fermement qu'il l'est affirmé dans l'ouvrage. Entre l'animal et l'homme, la communauté du vivant l'emporterait, en somme, sur la postulation de la différence humaine. Le problème est alors celui de savoir si l'ouverture de ces frontières, ou leur inexistence, relève d'une ontologie de l'intime conviction, vécue à l'intérieur de soi, éventuellement silencieuse, ou bien s'opère sur le mode intellectuel, donc réfléchi, à la manière dont le métaphysicien «fait» de l'ontologie, à froid si j'ose dire. Et s'ils avaient deux ontologies ou plus encore, dont seule l'ontologie publique, culturellement instituée, serait fermement dessinée? Le débat sur ce point me paraît être parfaitement légitime, non clos par décret d'autorité ethnographique.

CNRS-LAS-EHESS gerard.lenclud@wanadoo.fr 


$$
\text { 㧹 }
$$

